\title{
Guidance on the Use of Alternative Test Methods for the Safety Assessment of Cosmetics and Quasi-drugs
}

\author{
Hajime Kojima ${ }^{1(凶)}$, Yoshiaki Ikarashi ${ }^{2}$, Tokio Nakada ${ }^{3}$, \\ Akiko Yagami ${ }^{4}$, Kenji Sugibayashi ${ }^{5}$, Hiroaki Todo ${ }^{5}$, \\ Yukiko Hoshino $^{6}$, Naofumi Iizuka ${ }^{6}$, Takatoshi Nakamura ${ }^{6}$, \\ Shinichi Sekizawa ${ }^{6}$, Kazutoshi Shinoda ${ }^{6}$, Mio Yagi ${ }^{6}$, Daisuke Araki ${ }^{7}$, \\ Hitoshi Sakaguchi ${ }^{7}$, Hitoshi Sasa ${ }^{7}$, and Mariko Sugiyama ${ }^{7}$ \\ 1 Japanese Center for the Validation of Alternative Methods (JaCVAM), \\ National Institute of Health Sciences (NIHS), 3-25-26 Tonomachi, Kawasaki-Ku, \\ Kawasaki, Kanagawa 210-9501, Japan \\ h-kojima@nihs.go.jp \\ 2 National Institute of Health Sciences, Kawasaki, Kanagawa, Japan \\ ${ }^{3}$ Department of Dermatology, Showa University, Fujigaoka Hospital, \\ Yokohama, Japan \\ ${ }^{4}$ Department of Dermatology, Fujita Health University School of Medicine, \\ Toyoake, Aichi, Japan \\ ${ }^{5}$ Faculty of Pharmaceutical Sciences, Josai University, Sakado, Saitama, Japan \\ 6 Pharmaceuticals and Medical Devices Agency, Tokyo, Japan \\ 7 Japan Cosmetic Industry Association, Tokyo, Japan
}

\begin{abstract}
In accordance with a notification issued by Japan's Ministry of Health, Labour and Welfare (MHLW) in 2011, the Japanese Center for the Validation of Alternative Methods (JaCVAM) has accelerated applications for new test methods developed as alternatives to animal testing for the safety assessment of the manufacture and sales of cosmetic products and quasi-drug products that contain new ingredients. Taking advantage of this opportunity to strongly impact testing throughout Japan, researchers have been coordinating guidance on the use of such alternative test methods since 2012. Based on test guidelines issued by the Organisation for Economic Co-operation and Development (OECD) and JaCVAM evaluation documents, dermatologists and representatives of cosmetic companies as well as specialists from both the Pharmaceuticals and Medical Devices Agency (PMDA) and the National Institute of Health Sciences (NIHS) have drafted guidance documents for a number of alternative test methods.
\end{abstract}

Keywords: Cosmetic $\cdot$ Quasi-drug $\cdot \mathrm{JaCVAM} \cdot$ Guidance document

Presented at the First Asian Congress on Alternatives and Animal Use in the Life Sciences, Karatsu Civic Hall in Karatsu, Saga, Fukuoka, Japan on 16 Nov 2016.

(C) The Author(s) 2019

H. Kojima et al. (Eds.): Alternatives to Animal Testing, pp. 63-68, 2019.

https://doi.org/10.1007/978-981-13-2447-5_8 
A complete ban on the marketing of cosmetic products and cosmetic ingredients that have been tested on animals has been in effect in the EU since March 2013 [1]. In the light of this regulation, almost all Japanese cosmetic manufacturers have ceased the use of animal testing in the development of cosmetic products and ingredients. Just as in the EU, Japanese regulatory agencies as well as the MHLW have adopted positive and negative lists and generally do not require permits for the manufacture and sale of new cosmetic products that contain known ingredients. In cases where safety assessment of the manufacture and sales of quasi-drug products that contain new ingredients and new cosmetic ingredients is required, however, the MHLW still requires that applications for safety assessment be supported with animal data.

\section{MHLW Updates}

As shown in Table 1, the use of new cosmetic ingredients requires the submission of results from safety assessment performed with animal testing, In such cases, the test methods must ordinarily conform to notifications regarding pharmaceutical safety, OECD test guidelines, and other publicly-recognized standards. They must also appear in the keshohin-iyakubu gaihin seizou hanbai gaidobukku 2011-2012 (the 2011-2012 Guidebook on the Manufacture and Sales of Cosmetics and Quasi-Drugs) [2]. Also, all animal testing must conform to koseirodousho non shokan suru jisshikikan ni okeru doubutsu jiken tou no jisshi ni kan suru kihonhoushin (Basic Policies Regarding Implementation of Animal and Other Testing at Organizations under the Jurisdiction of the MHLW), as well as all other laws and regulations relevant to animal and other testing [3].

An administrative notice [4] issued in July 2006 by the MHLW Evaluation and Licensing Division indicated that the inclusion of results from alternative test methods as documentary evidence in an application was acceptable, provided that the alternative test methods conformed to accepted OECD test methods or had been similarly validated in a suitable manner. The administrative notice further stipulated that, in cases where animal testing was to be performed, if information about the physicochemical properties of the test chemical itself or similar chemical substances as well as the results of non-animal testing (in vitro, in chemico, in silico testing) led to the reasonable expectation that the test chemicals could induce distress or suffering in test animals, efforts to mitigate such distress or suffering by diluting test chemicals would be required.

On 4 February 2011, the MHLW issued a notification that data obtained with alternative test methods approved by JaCVAM could be used for the submission of quasi-drug applications, or for petitions to include ingredients in the Standards for Cosmetics [5]. Information is available on the JaCVAM website [6] to help ensure proper preparation of the documentary evidence to be included with applications for approval of manufacturing and sales of quasi-drug products as well as in requests for revisions to the positive list for cosmetics. This information has been made available to the appropriate businesses and other concerned parties industrywide. In addition, administrative notices issued in April 2012 and May 2013 by the MHLW Evaluation and Licensing Division publicized the availability of alternative test methods for 
Table 1. Test methods for regulatory use in safety assessment of ingredients in Japan

\begin{tabular}{l|l}
\hline Quasi-drug safety evaluation guidance & \\
\hline For main ingredients & For additives \\
\hline Acute toxicity & Acute oral toxicity \\
\hline Repeat dose toxicity (Sub-chronic, Chronic) & NR \\
\hline Reproductive toxicity & NR \\
\hline Local irritation (Skin, Mucous membrane) & Primary skin irritation \\
\cline { 2 - 2 } & Cumulative skin irritation \\
\cline { 2 - 2 } & Ocular irritation \\
\hline Skin sensitization & Skin sensitization \\
\hline Photo safety & Photo safety \\
\hline Genotoxicity & Genotoxicity \\
\hline Carcinogenicity (case by case) & NR \\
\hline ADME & ADME (case by case) \\
\hline Human patch test & Human patch test \\
\hline Human use test & NR \\
\hline
\end{tabular}

NR: Not Required

phototoxicity testing and skin sensitization testing for use in safety assessment of cosmetics and quasi-drugs [5]. Additional information on other alternatives to animal testing will be issued as it becomes available. Dermatologists and representatives of cosmetic companies as well as specialists from both the PMDA and the NIHS have drafted guidance documents for a number of alternative test methods based on OECD test guidelines and JaCVAM evaluation documents.

Alternative test methods developed for OECD test guidelines are useful in predicting potential hazards of exposure to chemicals, even though they are not always suitable for providing the wide range of information needed for comprehensive safety and risk assessment. Thus, manufacturers often have difficulty gaining approval from Japanese regulators for new cosmetic ingredients unless they perform animal testing [6].

\section{JaCVAM Update}

JaCVAM was established to promote the use of alternative methods to animal testing in regulatory studies, thereby replacing, reducing, or refining the use of animals wherever possible, while meeting the responsibility of the National Center for Biological Safety and Research (BSRC) to ensure the protection of the public by assessing the safety of chemicals and other materials, as stipulated in the regulations of the NIHS in November 2005. JaCVAM activities are also beneficial to the application and approval for the manufacture and sale of pharmaceutical, chemical, pesticide and other products, as well as to revisions to standards for cosmetic and quasi-drug products [7-9].

Over the past 12 years, JaCVAM has played an active role in gaining approval for the adoption of OECD test guidelines for more than ten different test methods 
developed by Japanese researchers [6] and has evaluated alternative methods to animal testing based on OECD test guidelines as well as International Conference on Harmonisation of Technical Requirements for Registration of Pharmaceuticals for Human Use (ICH) guidelines. Moreover, JaCVAM has recommended twenty-one test methods for adoption by Japanese regulatory agencies [7], as shown in Table 2. The notification issued by MHLW in 2011 presented JaCVAM with the opportunity to impart a strong impact on testing throughout Japan by accelerating the development of new non-animal test methods.

\section{Guidance for Alternative to Animal Testing}

The following guidance documents have been approved by the MHLW [5]. Based on OECD test guidelines and JaCVAM evaluation documents, dermatologists and representatives of cosmetic companies as well as specialists from both the PMDA and the NIHS have drafted guidance documents for a number of alternative test methods.

- Guidance on the use of alternative test methods for skin-sensitization and phototoxicity in safety assessment of cosmetics and quasi-drugs (Appendix 1: Guidance on the use of $\mathrm{LLNA}^{1}$ for skin sensitisation test as an alternative test method in safety assessments of cosmetics and quasi-drugs, Appendix 2: Guidance on the use of the in vitro $3 \mathrm{~T} 3 \mathrm{NRU}^{2}$ phototoxicity test as an alternative test method in safety assessments of cosmetics and quasi-drugs), dated April 26, 2012

- Guidance on the use of alternative test methods for skin-sensitization (LLNA:DA, LLNA:BrdU-ELISA) in safety assessments of cosmetics and quasi-drugs, dated May 30, 2013,

- Guidance on the use of the $\mathrm{BCOP}^{3}$ test as an alternative method for testing ocular irritation in the safety assessment of cosmetics and quasi-drugs, dated February 4, 2014

- Points of consideration for ocular irritation testing in the safety assessment of cosmetics and quasi-drug, dated February 27, 2015

- Guidance on the use of the $\mathrm{ICE}^{4}$ test as an alternative method for testing ocular irritation in the safety assessment of cosmetics and quasi-drugs, dated November 16,2015

- Guidance on the use of in vitro skin penetration assay (in vitro skin absorption assay) in the safety assessment of cosmetics and quasi-drugs, dated November 15 , 2016

- Guidance on the use of combinations of in vitro skin sensitization assays in the safety assessment of cosmetics and quasi-drugs, dated January 2018.

\footnotetext{
${ }^{1}$ LLNA: Local lymph Node Assay.

2 NRU: Neutral Red Uptake.

${ }^{3}$ BCOP: Bovine Corneal Opacity and Permeability.

${ }^{4}$ ICE: Isolated Chicken Eye.
} 
Table 2. Test methods proposed to the Ministry of Health, Labour and Welfare by JaCVAM

\begin{tabular}{|c|c|}
\hline No. & Test Method \\
\hline 1 & $\begin{array}{l}\text { In vitro skin corrosion testing: i.e. Human Skin Model Test: Vitrolife-Skin, EpiDerm, } \\
\text { Episkin, SKinEthics, EpiCS }\end{array}$ \\
\hline 2 & In vitro skin corrosion testing: Transcutaneous Electrical Resistance Test Method (TER) \\
\hline 3 & In vitro skin corrosion testing: In Vitro membrane barrier test method for skin corrosion \\
\hline 4 & $\begin{array}{l}\text { In vitro skin irritation testing: i.e. Reconstructed Human Epidermis Test Method, Episkin, } \\
\text { Epiderm, SkinEthics and LabCyte EPI-Model }\end{array}$ \\
\hline 5 & $\begin{array}{l}\text { Bovine corneal opacity and permeability (BCOP) test for identifying ocular corrosives } \\
\text { and severe irritant }\end{array}$ \\
\hline 6 & Isolated chicken eye (ICE) test for identifying ocular corrosives and severe irritants \\
\hline 7 & Fluorescein leakage (FL) assay for identifying ocular corrosives and severe irritants \\
\hline 8 & Short Time Exposure In Vitro Test Method for assessing ocular irritation \\
\hline 9 & Reconstructed human Cornea-like Epithelium Eye Irritation Test Method \\
\hline 10 & Acute Eye irritation/Corrosion \\
\hline 11 & $\begin{array}{l}\text { Local lymph node assay (LLNA) and reduced LLNA for identifying skin sensitising } \\
\text { substances }\end{array}$ \\
\hline 12 & $\begin{array}{l}\text { LLNA:DA, a non-radioactive modification of the LLNA method, which quantifies } \\
\text { adenosine triphosphate (ATP) content via bioluminescence, as an indicator of } \\
\text { lymphocyte proliferation for identifying skin sensitising substances }\end{array}$ \\
\hline 13 & $\begin{array}{l}\text { LLNA:BrdU-enzyme linked immunosorbent assay (ELISA), a non-radioactive } \\
\text { modification of the LLNA method, which utilises non-radiolabelled 5-bromo-2- } \\
\text { deoxyuridine (BrdU) in an ELISA-based test system, to measure lymphocyte } \\
\text { proliferation for identifying skin sensitising substances }\end{array}$ \\
\hline 14 & Direct Peptide Reactivity Assay for skin sensitisation Testing \\
\hline 15 & ARE-Nrf2 Luciferase Test Method assay for skin sensitisation testing \\
\hline 16 & h-CLAT (human Cell Line Activation Test) for skin sensitisation testing \\
\hline 17 & Skin absorption in vitro method \\
\hline 18 & Reactive Oxygen Species (ROS) assay for photosafety assessment \\
\hline 19 & In vitro cytotoxicity test for estimating starting doses for acute oral systemic toxicity tests \\
\hline 20 & $\begin{array}{l}\text { BG1Luc estrogen receptor transactivation assay (ETRA), for identifying oestrogen } \\
\text { receptor agonists and antagonists }\end{array}$ \\
\hline 21 & Stably transfected Transcriptional Activation Assay to Detect ER mediated activity \\
\hline
\end{tabular}

Almost all Japanese cosmetic companies have stopped animal testing voluntarily and now hope that the MHLW and PMDA will approve the use of new cosmetic ingredients without animal testing. Many procedures for regulatory acceptance still require animal testing, and it is difficult to gain approval even for additives to quasidrug products without data from animal testing. Given the current situation, JaCVAM has attempted to promote the development of guidance for the use of alternatives to animal testing. Unfortunately, there are still many types of testing for which nonanimal test methods are limited. In particular, there are as of yet no suitable non-animal 
test methods available as alternatives to the LLNA, LLNA:DA, LLNA:BrdU-ELISA, or Draize eye test, and the time has come to accelerate the development of and guidance for the use of in vitro test methods for new cosmetic ingredients.

\section{References}

1. EC (2004) Time tables for the phasing-out of animal testing in the framework of the 7th amendment to the cosmetics directive (Council Directive 76/768/EEC). Commission Staff Working Documents, SEC82004)1210

2. MHLW (2011) Guidebook on the manufacture and sales of cosmetics and quasi-drugs [In Japanese]. Yakuji Nippo Ltd, Tokyo, Japan, pp 159-182

3. MHLW (2005) Guide for the care and use of laboratory animals (in Japanese). Ministry of Health, Labour and Welfare, Tokyo, Japan

4. MHLW (2006) Cosmetics standard amendment request and manufacturing and marketing approval of quasi-drugs (in Japanese). Ministry of Health, Labour and Welfare, Tokyo, Japan

5. PMDA (2018) Homepage (in Japanese). Pharmaceuticals and Medical Devices Agency, Tokyo, Japan. https://www.pmda.go.jp/review-services/drug-reviews/about-reviews/q-drugs/ 0002.html

6. OECD (2018) Test guideline. Organisation for Economic Co-operation and Development, Paris, France. http://www.oecd-ilibrary.org/environment/oecd-guidelines-for-the-testing-ofchemicals-section-4-health-effects_20745788

7. JaCVAM (2018) Homepage. Japanese Center for the Validation of Alternative Methods, Tokyo, Japan. http://www.jacvam.jp/en/index.html

8. Barroso J, Ahn IY, Caldeira C et al (2016) Validation of alternative methods for toxicity testing. Adv Exp Med Biol 856:343-386

9. Kojima H (2013) Update from the Japanese center for the validation of alternative methods (JaCVAM). ATLA 41:435-441

Open Access This chapter is licensed under the terms of the Creative Commons Attribution 4.0 International License (http://creativecommons.org/licenses/by/4.0/), which permits use, sharing, adaptation, distribution and reproduction in any medium or format, as long as you give appropriate credit to the original author(s) and the source, provide a link to the Creative Commons licence and indicate if changes were made.

The images or other third party material in this chapter are included in the chapter's Creative Commons licence, unless indicated otherwise in a credit line to the material. If material is not included in the chapter's Creative Commons licence and your intended use is not permitted by statutory regulation or exceeds the permitted use, you will need to obtain permission directly from the copyright holder.

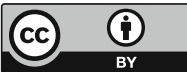

